\title{
Load-dependent Variable Gearing Mechanism of Muscle-like Soft Actuator
}

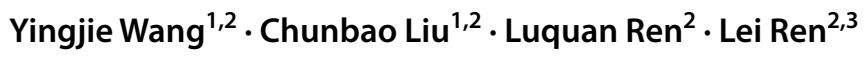

Received: 11 May 2021 / Revised: 11 November 2021 / Accepted: 16 November 2021 / Published online: 16 December 2021

(c) The Author(s) 2021

\begin{abstract}
Pennate muscle is characterized by muscle fibers that are oriented at a certain angle (pennation angle) relative to the muscle's line of action and rotation during contraction. This fiber rotation amplifies the shortening velocity of muscle, to match loading conditions without any control system. This unique variable gearing mechanism, which characterized by Architecture Gear Ratio (AGR), is involves complex interaction among three key elements: muscle fibers, connective tissue, and the pennation angle. However, how three elements determine the AGR of muscle-like actuator is still unknown. This study introduces a Himisk actuator that arranges five contractile units at a certain pennation angle in a flexible matrix, the experiment and simulation results demonstrated that the proposed actuator could vary AGR automatically in response to variable loading conditions. Based on this actuator, we present a series of actuators by simulations with the varying pennation angle $(P)$, elastic modulus of the flexible matrix $(E)$, and number of contractile units $(N)$ to analyze their effects on AGR, and their interaction by three-factor analysis of variance. The results demonstrated that $P$ and $N$ effect on the AGR significantly, while $E$ effects on AGR slightly, which supported the idea that the $P$ is the essential factor for the AGR, and $N$ is also an important factor due to the capability of force generation. This provides a better understanding of mechanical behavior and an effective optimizing strategy to muscle-like soft actuator.
\end{abstract}

Keywords Bioinspired $\cdot$ Actuator $\cdot$ Pennate muscle $\cdot$ Gearing mechanism

\section{Introduction}

Skeletal muscle has been deemed a "typical soft actuator" due to their deformable characteristics [1]. In pennate muscle, the muscle fibers are oriented at an angle relative to the muscle's line of action and rotation during contraction [2]. The rotation of muscle fibers increases the whole muscle shortening. The displacement or velocity amplification of fiber shortening can be characterized by the muscle's Architectural Gear Ratio (AGR), which is defined as the ratio of

Chunbao Liu

liuchunbao@jlu.edu.cn

Lei Ren

lei.ren@manchester.ac.uk

1 School of Mechanical and Aerospace Engineering, Jilin University, Changchun 130022, China

2 Key Laboratory of Bionic Engineering, Ministry of Education, Jilin University, Changchun 130022, China

3 School of Mechanical, Aerospace and Civil Engineering, University of Manchester, Manchester M13 9PL, UK muscle shortening (or muscle velocity) to fiber shortening (or fiber velocity) [3]. The range of AGR depends on the load conditions. When contracting against light loads, the pennate angle of muscle fibers undergo significant changes. Conversely, when contracting against heavy loads, muscle fibers rotate less and muscle thickness and displacement decreases, which indicates that pennate muscles act as a "automatic transmission system" which is an important mechanical feature of skeletal muscle.

In recent years, a variety of soft actuators have been developed with the aim to replicate mechanical features; for example, dielectric elastomers [4-7], shape-memory alloys [8-10], fiber-based and polymer carbon artificial muscles [11-13], and fluidic actuators [14-20]. All artificial actuators demonstrated here can achieve linear contraction, high strain/stress, and intrinsic mechanical properties. However, to date, there are few studies that provide insight into the variable gearing property. Of these, McKibben artificial muscles $[21,22]$ is a most prevalent fluidic actuator, because they can expand radially when they shorten. This change is a critical feature of the variable gearing mechanism of 
soft actuators. Therefore, lots of researchers have designed skeletal muscle-like actuators based on McKibben artificial muscles. For example, Azizi et al. [14] created a pneumatic actuator by paralleling five McKibben artificial muscles in an array. Similar to skeletal muscle, the pennate array of McKibben is well demonstrated to be a load-dependent variable gearing mechanism. However, this design lacks the complex architectural features, such as the connective tissue, which will alter the deformation mechanism of actuator, thus to influence the gear ratio. There is still a significant gap between artificial muscle and biological skeletal muscle. The interaction of contractile units, pennation angle, and flexible behavior of connective tissue remains unknown. Roche et al. $[15,18]$ reported a pneumatic artificial heart by embedding contractile units into a flexible matrix to achieve a heart-like rotational motion. Actuation of the myocardium is different from skeletal muscle but provides a valuable reference for the design of skeletal muscle, as natural muscle actuation can be achieved by arranging muscle fibers parallel within the connective tissue [2, 3, 23].

Here, we introduced a Himisk actuator that arranged McKibben-based contractile units (MFU) in a flexible matrix with a pennation angle. MFU acts as a muscle fiber that produces an active force, and a flexible matrix acts as connective tissue that produced passive deformation due to its elastic behavior. To investigate how the MFU and flexible matrix effect on the variable gearing performance of actuator, we proposed three soft actuators (PA30-Matrix, PA30-NoMatrix, and PA0-Matrix). The results demonstrate that actuators can vary the muscle shape, pennation angle and AGR automatically in response to variable loading conditions, similar to skeletal muscle [2], and the output work (power) of the pennate actuator can be amplified, when AGR $>1$. Based on the PA30-Matrix actuators, to evaluate how the pennation angle $(P)$, elastic modulus of the flexible matrix $(E)$, and number of muscle fibers $(N)$ effect on AGR, we performed a series of actuators using variable $P, E$, and $N$ based on simulation and performed three-factor analysis of variance (ANOVA). The results demonstrate that the $P$ and $N$ effect on AGR significantly, while the $E$ had a negligible effect. The results supported the idea that the $P$ is the most important factor for the variable gearing mechanism, while $N$ is also important due to its capability of force generation $[24,25]$. This provides a better understanding of mechanical behavior and an effective optimizing strategy to muscle-like soft actuator.

\section{Bio-inspired Design}

Skeletal muscles are composed of muscle fibers and connective tissue. The fibers are oriented at an angle relative to the muscle's line of action (Fig. 1a). When muscle fibers are activated, they shorten in length and increase in diameters; these changes must be accommodated by increase in one or more orthogonal direction (thickness, width, or both directions). This will cause muscle contraction due to its constant volume. There is a complex interaction between muscle fiber and connective tissue during contraction [23, 26]. As depicted in Fig. 1b, the muscle fibers are oriented at an angle relative to the line of force and becoming more oblique as they shorten. The rotation of fibers increases the muscle shortening. The advantage can be characterized as the AGR, quantified as muscle shortening (or muscle velocity)/fiber shortening (or fiber velocity). The muscle operates in a high AGR, pennation angle, displacement and thickness, during rapid contractions. And with a low AGR, pennation angle, displacement and thickness, during forceful contractions. The muscle fiber force, pennate angle, and behaviours of connective tissue are the main factors affecting muscle deformation and AGR [27].

Inspired by pennate muscle, we developed three soft actuators, named PA30-Matrix, PA0-Matrix, and PA30-NoMatrix. As shown in Fig. 1c, these include $0^{\circ}$ and $30^{\circ}$ pennation angle arrangements with a flexible matrix, and a $30^{\circ}$ pennation angle configuration without the matrix fabricated by pouring. The physical model of three type soft actuators is shown in Fig. 1d. This allows us to investigate the effect of MFUs and flexible matrix on the actuator's performance.

\section{Methods}

\subsection{Numerical Simulation Methods}

The simulations were performed using ABAQUS 2019 (Simulia, USA)/Standard. We used a fluid cavity interaction property and rebar layers to model the motion of MFU. The model of MFUs and flexible matrix were assumed to be isotropic with linear elastic material properties. The contractile units (Young's modulus 1.5 MPa, Poisson's ratio 0.31) [18], double symmetrical rebar layers (Young's modulus $100 \mathrm{MPa}$, Poisson's ratio 0.3) [28] with linear elastic material, and the flexible matrix with Young's modulus of 0.6 MPa and Poisson's ratio of 0.475 [28]. The interaction between the contractile units and the flexible matrix was defined as a tie constraint; the inflation behavior of the contractile units was simulated with a fluid cavity; and the applied pressure was the same as that of the pressure during the experiment. For the PA0-Matrix actuator, the MFUs and the flexible matrix were meshed using a total number of 1208 and 16,277, respectively. For PA30-NoMatrix, the MFUs and the two fix-sides were meshed using a total number of 1870 and 8158, respectively. For PA30-Matrix, the MFUs and the flexible matrix were meshed using a total number of 1183 and 14,700 quadratic tetrahedral (C3D10) 
Fig. 1 Structure of the skeletal muscle and soft actuator. a Contraction of a unipennate muscle and the architectural gear ratio measured. b Muscle as a composite, hierarchical structure [23]. C Soft actuators of PA30Matrix, PA30-NoMatrix, and PA0-Matrix

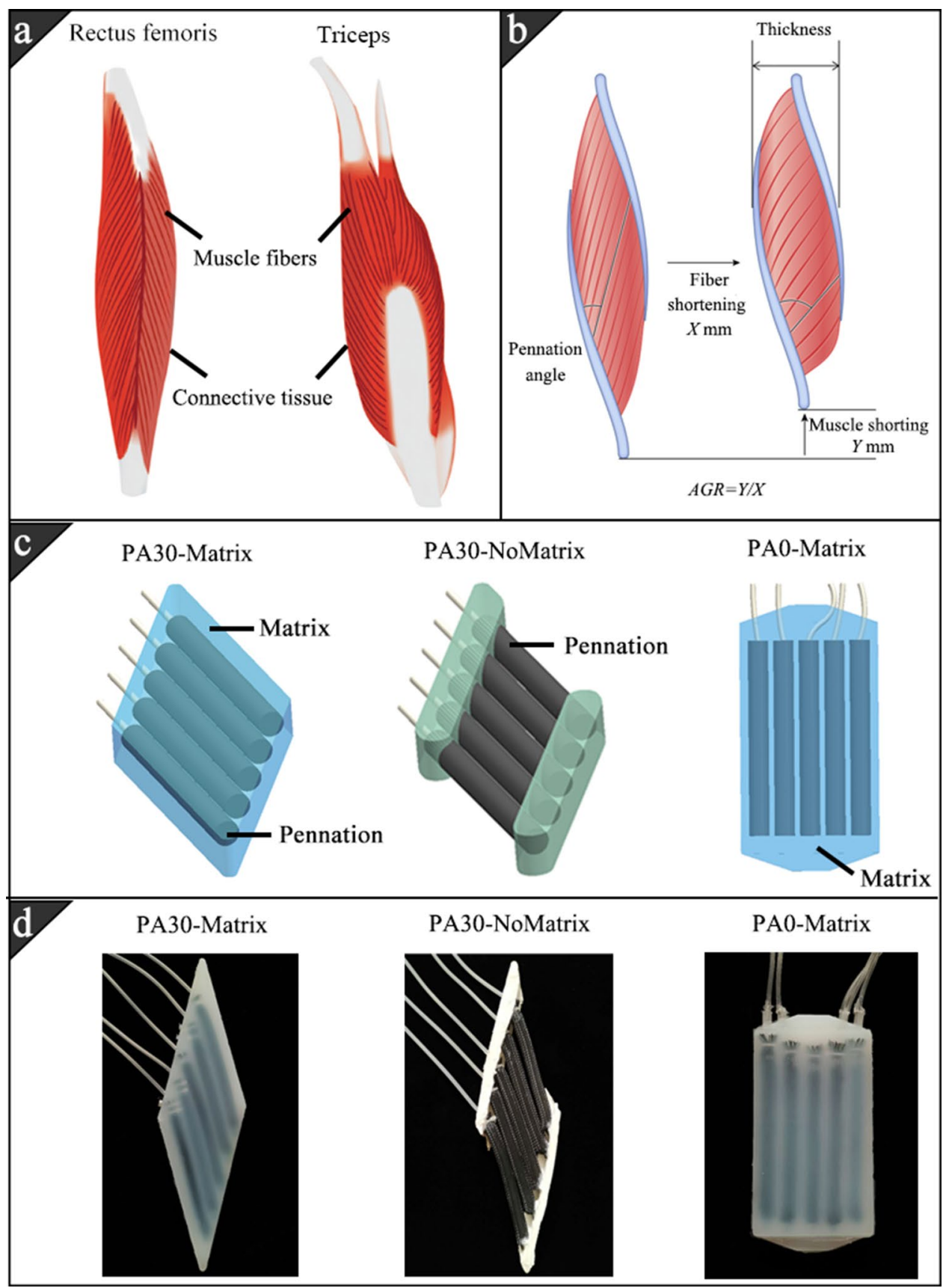

elements, respectively. All of the mesh for three actuators were determined through a convergence analysis by gradually increasing the mesh density until the deviations in the estimated displacement reached within 5\%. The deformations, stress, and strain distributions at the maximum pressure of 0.2 MPa were simulated.

Based on the PA30-Matrix actuator's configuration and parameter $\left(P=30^{\circ}, N=5, E=0.6 \mathrm{MPa}\right)$, we analyzed the variable gearing mechanism with variable $P, E$, and $N$ under different loads, as shown in Table 1. To further analyze the interaction of $E(0.5,0.6,0.7$, and $0.8 \mathrm{MPa}), P\left(25^{\circ}, 30^{\circ}\right.$, $\left.35^{\circ}, 40^{\circ}\right)$ and $N(3,4,5,6)$ and their effect on AGR, we
Table 1 AGR, $\Delta L_{\text {muscle }}, \Delta L_{\mathrm{mfu}}$ at different $P, N$, and $E$ under different loads

\begin{tabular}{|c|c|c|}
\hline \\
\hline \multicolumn{3}{|l|}{$P=30^{\circ}, N=5$} \\
\hline$E=0.5,0.6,0.7 \mathrm{MPa}$ & $P=25^{\circ}, 30^{\circ}, 35^{\circ}, 40^{\circ}$ & $N=3,4,5,6$ \\
\hline
\end{tabular}

performed 64 sets of numerical simulations under zero load at $0.2 \mathrm{MPa}$, as shown in Table 2. $\left(P_{25}, P_{30}, P_{35}, P_{40}\right.$ represent $P=25^{\circ}, P=30^{\circ}, P=35^{\circ}, P=40^{\circ} ; N_{3}, N_{4}, N_{5}, N_{6}$ represent 
Table 2 AGR at different $P, N$, and $E$ under zero load at $0.2 \mathrm{MPa}$

\begin{tabular}{|c|c|c|c|c|c|c|c|c|c|c|c|c|c|c|c|c|}
\hline & $P_{25}$ & $P_{30}$ & $P_{35}$ & $P_{40}$ & $P_{25}$ & $P_{30}$ & $P_{35}$ & $P_{40}$ & $P_{25}$ & $P_{30}$ & $P_{35}$ & $P_{40}$ & $P_{25}$ & $P_{30}$ & $P_{35}$ & $P_{40}$ \\
\hline$E(\mathrm{MPa})$ & $N_{3}$ & & & & $N_{4}$ & & & & $N_{5}$ & & & & $N_{6}$ & & & \\
\hline$E_{05}$ & 1.89 & 1.48 & 1.32 & 1.22 & 1.65 & 1.39 & 1.25 & 1.16 & 1.45 & 1.35 & 1.31 & 1.13 & 1.66 & 1.37 & 1.23 & 1.15 \\
\hline$E_{06}$ & 1.92 & 1.48 & 1.33 & 1.23 & 1.64 & 1.39 & 1.25 & 1.16 & 1.45 & 1.34 & 1.30 & 1.10 & 1.65 & 1.36 & 1.22 & 1.14 \\
\hline$E_{07}$ & 1.94 & 1.48 & 1.33 & 1.23 & 1.63 & 1.39 & 1.25 & 1.16 & 1.45 & 1.33 & 1.30 & 1.11 & 1.65 & 1.36 & 1.22 & 1.13 \\
\hline$E_{08}$ & 1.96 & 1.48 & 1.33 & 1.23 & 1.62 & 1.39 & 1.25 & 1.15 & 1.48 & 1.36 & 1.31 & 1.11 & 1.64 & 1.36 & 1.21 & 1.12 \\
\hline
\end{tabular}

$N=3, N=4, N=5, N=6 ; E_{05}, E_{06}, E_{07}, E_{08}$ represent $E=0.5$, $E=0.6, E=0.7, E=0.8$ ).

\subsubsection{Measures of Displacement, Pennation Angle, and Thickness}

As shown in Fig. 2a, we set the middle point (point 1, point 3 ), and the vertex point (point 2) before the simulation to quantify the changes of displacement, pennation angle, and thickness of the PA30-Matrix actuator. The displacement of actuator was defined as a vertical contraction of soft actuator. The thickness was determined by measuring the distance between the leftmost and rightmost vertex of the actuator. The acute angle between the two adjacent edges of the actuator was measured as the pennate angle $(\theta)$. We measured the angle by linking point 1 , point 2 , and point 3 when actuator in stable. The measurement of displacement, pennation angle, and thickness of the PA30-NoMatrix actuator was similar to those of the PA30-Matrix actuator. However, there was a small difference at the pennation angle measurement due to it lacking a flexible matrix for reference. We obtained the angle ( $\left.\theta^{\prime}\right)$ at three points, of which point 2 was the intersection of the left edge and centerline of the contractile unit, while points 1 and 3 was on the left edge and centerline respectively, as shown in Fig. 2b. The measurement of PA0-Matrix is shown in Fig. 2c. The PA30-Matrix, PA30-NoMatrix and PA0-Matrix actuators were tested in experiment, as shown in Fig. 3. An S-type force sensor was used to measure the actuator force, and a laser displacement sensor was used to record the displacement. The force data were collected by a DYB-5 dynamic strain gauge, and then transmit to VIBSYS vibration signal acquisition and analysis equipment. To obtain the changes of pennation angle and thickness, a digital camera was used to record the actuator behaviour. We selected two key frames corresponding to the instant before the load off the surface and also the instant when the contraction reached to stable. The two images were measured for the thickness and pennation angle.

\subsubsection{Measures of AGR}

The AGR of the pennate muscle was calculated as the ratio of whole muscle shortening to fiber shortening [3], or the muscle velocity to the fiber velocity during isotonic contraction [2]. We defined the AGR as the ration of actuator longitudinal shortening ( $\Delta L_{\text {actuator }}$ ) to contractile unit

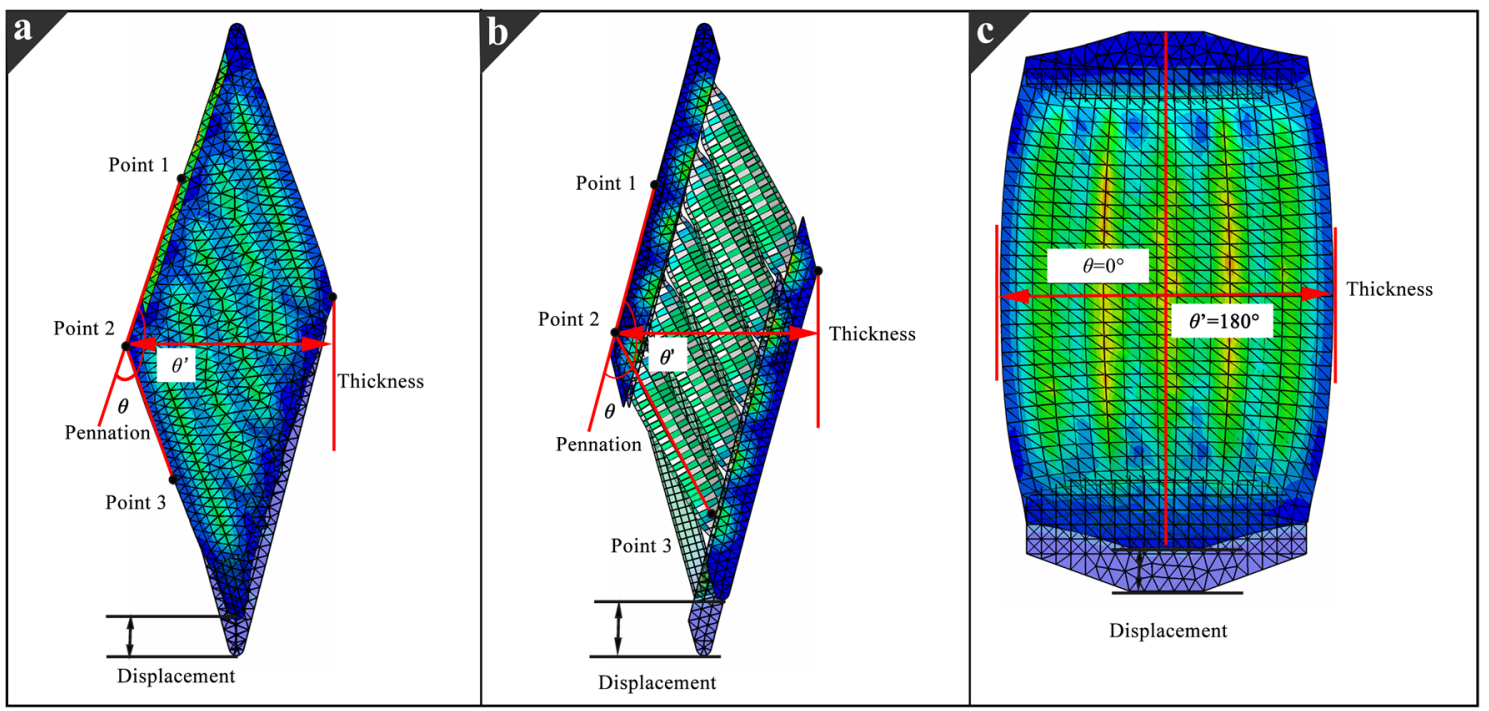

Fig. 2 Measurement of displacement, thickness, and pennation of a PA30-Matrix, b PA30-NoMatrix, and c PA0-Matrix actuators 


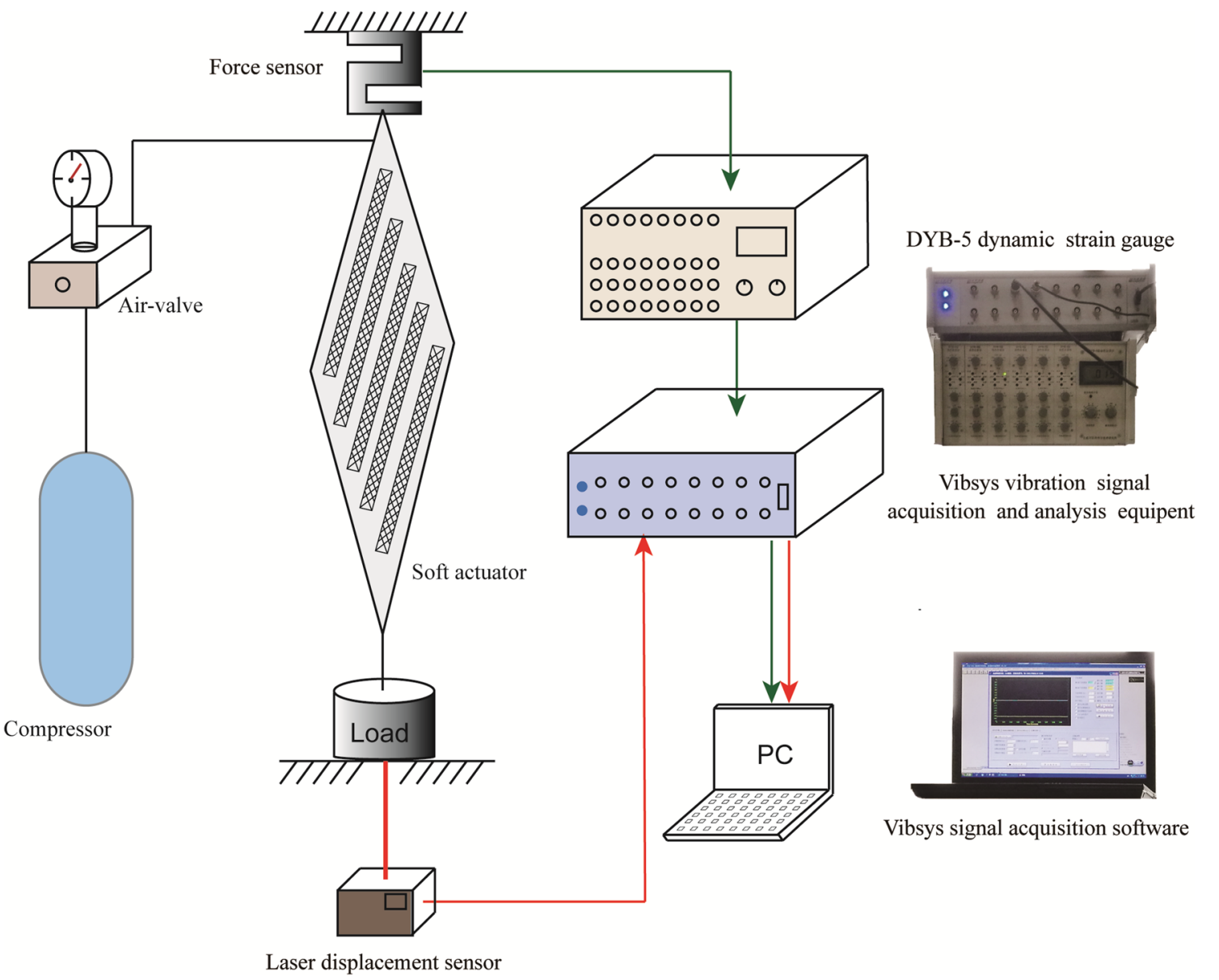

Fig. 3 Experimental installation for measurement of actuator

shortening $\left(\Delta L_{\mathrm{mfu}}\right)$ based on the standard finite element model. $\Delta L_{\text {actuator }}$ was defined as vertical displacement, and $\Delta L_{\mathrm{mfu}}$ was the relative displacement between the two center points of the two ends of the middle contractile unit.

\subsection{Data Analyses}

All data analyses were performed using the IBM Statistical Package for the Social Sciences (SPSS) Statistics software version 26 (SPSS Inc., Chicago, IL, USA). Three-way ANOVA was used to test the significance of the $P, N$, and $E$ for the AGR under the conditions of $P$ at $25^{\circ}, 30^{\circ}, 35^{\circ}$, and $40^{\circ} ; N$ at $3,4,5$, and 6 ; and $E$ at $0.5 \mathrm{MPa}, 0.6 \mathrm{MPa}, 0.7 \mathrm{MPa}$, and $0.8 \mathrm{MPa}$, respectively. The valid numbers for the simulations analyzed are shown in Table 2. Statistical significance was set at $p<0.05$.

\section{Results}

\subsection{Deformation of Three Actuators}

As shown in Fig. 4, the model predicted load-dependent changes of actuators during experiment (Fig. 4a) and simulation (Fig.4b, c). The deformation of soft actuators under different loads are shown in Fig. 4b. As expected, the MFUs contracted in length and expanded in diameter when air pressure was applied, this resulted in deformation of soft actuator. Compared with the test (Fig. 4a) and simulation (Fig. 4b), three actuators show similar deformations. In addition, the displacements and thickness all decreased with the loads increasing. The stress of the three actuators was depicted in Fig. 4c. When at low-loads, the increased pennation angle and small passive stretch of the flexible matrix 


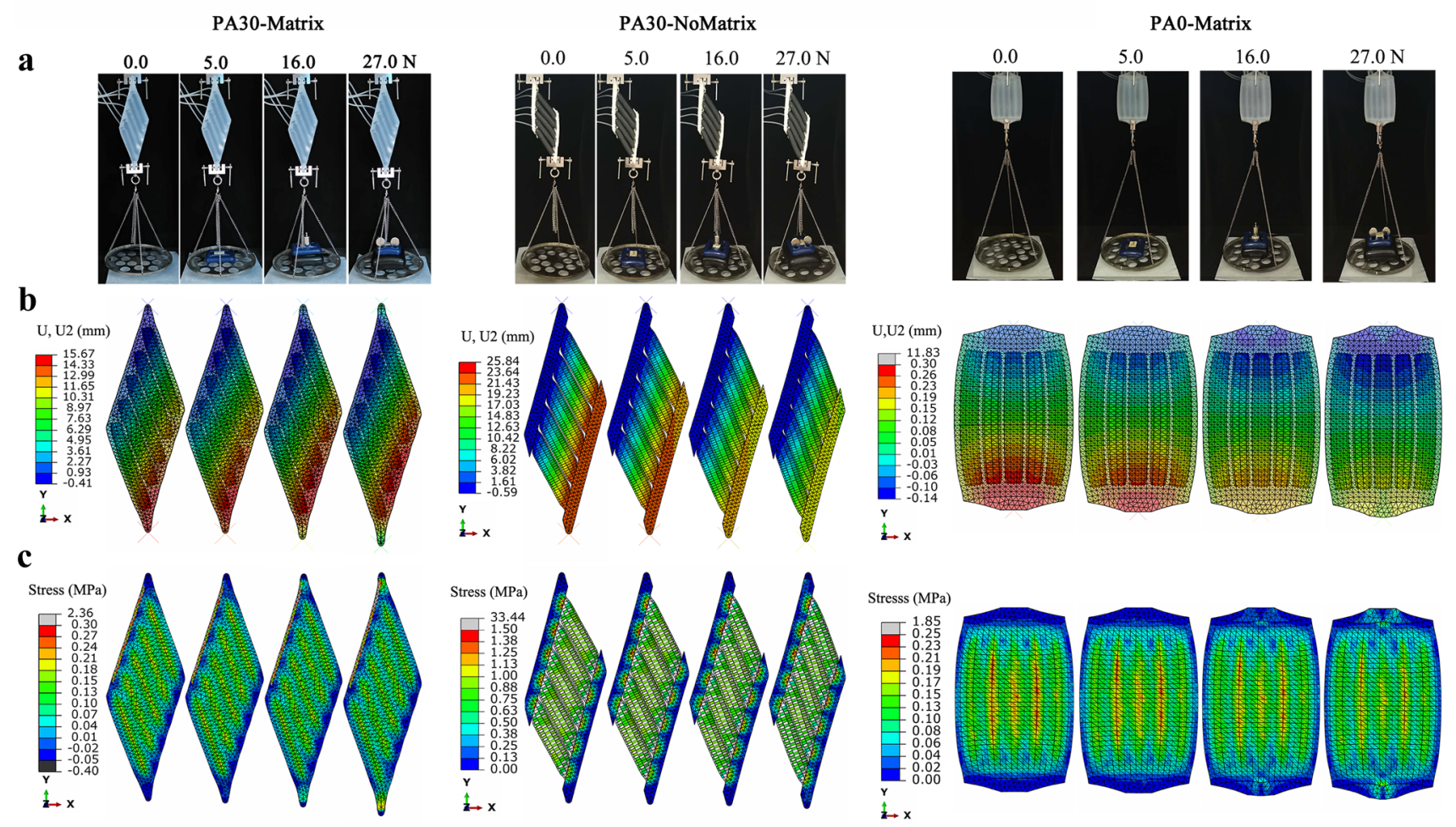

Fig. 4 Finite element simulations of the PA0-Matrix, PA30-Matrix, and PA30-NoMatrix actuators with varying application loads. a The recorded deformations of actuators when acting against the increased

tended to increase the actuator thickness and displacement. At high-load contraction, the decreased pennation angle and large passive stretch of the flexible matrix decreased thickness and displacement. There are notably tensile deformations present in the PA30-Matrix and PA0-Matrix actuators at the position where the loads were applied. The PA30NoMatrix actuator has no obvious tensile deformations due to the rigid connecting. The results demonstrated that the finite element model can clearly reveal the deformation and driving mechanism of the soft actuator.

To further verify the deformation of three actuators, we quantified the displacement, pennation angle, and thickness as shown in Fig. 5. Large displacement of actuators was generated at low loads, and small displacements were generated at high loads (Fig. 5a-c). The predicted pennation angle (Fig. 5d-f) and thickness (Fig. 5g-i) decreased as the loads increased as well. Load-dependent variation of changes in thickness and pennation angle resulted in a load-dependent AGR. The AGR decreased significantly as the load increasing, which the trend is similar to the biological muscle and the experiment (Fig. 5j, k). However, there were disagreements between the Finite Element Analysis (FEA) and the load $(0,5,16$, and $27 \mathrm{~N})$ at an air pressure of $0.2 \mathrm{MPa}$. b Vertical displacement. $\mathbf{c}$ von Mises stress

experiment for PA30-Matrix actuator (Fig. 5j). In simulations, deformation of the rebar layer is determined from the deformation gradient of the underlying shell. However, in experiment, when the woven mesh changed their orientation during contraction, its deformation is not only determined from the deformation of the underlying flexible tube but the frictional resistance from flexible matrix and flexible tube as well. The additional friction will limit the shortening of MFU in experiment lower that in the simulation. In addition, the $\Delta L_{\text {actuator }}$ in simulation is lower than the experiment, the displacement of actuator at zero loads is about $14.9 \mathrm{~mm}$, which is lower than the experiment $(17.0 \mathrm{~mm})$. In the paper, the AGR of the soft actuator was calculated as the ratio of $\Delta L_{\text {actuator }}$ to $\Delta L_{\mathrm{mfu}}$. For the PA30-matrix actuator, its $\Delta L_{\text {actuator }}$ in simulation is lower than the experiment. Therefore, the accumulative error contributes to the large relative error. However, the relative error was not influenced the tendency of deformation (displacement, thickness, pennation angle) and AGR, all of them decreased with increasing loads, similar to skeletal muscle. And it does not affect the judgment of the variation tendency. The AGR of the PA30Matrix was maximum $(\mathrm{AGR}=1.4)$ and had a wide speed 

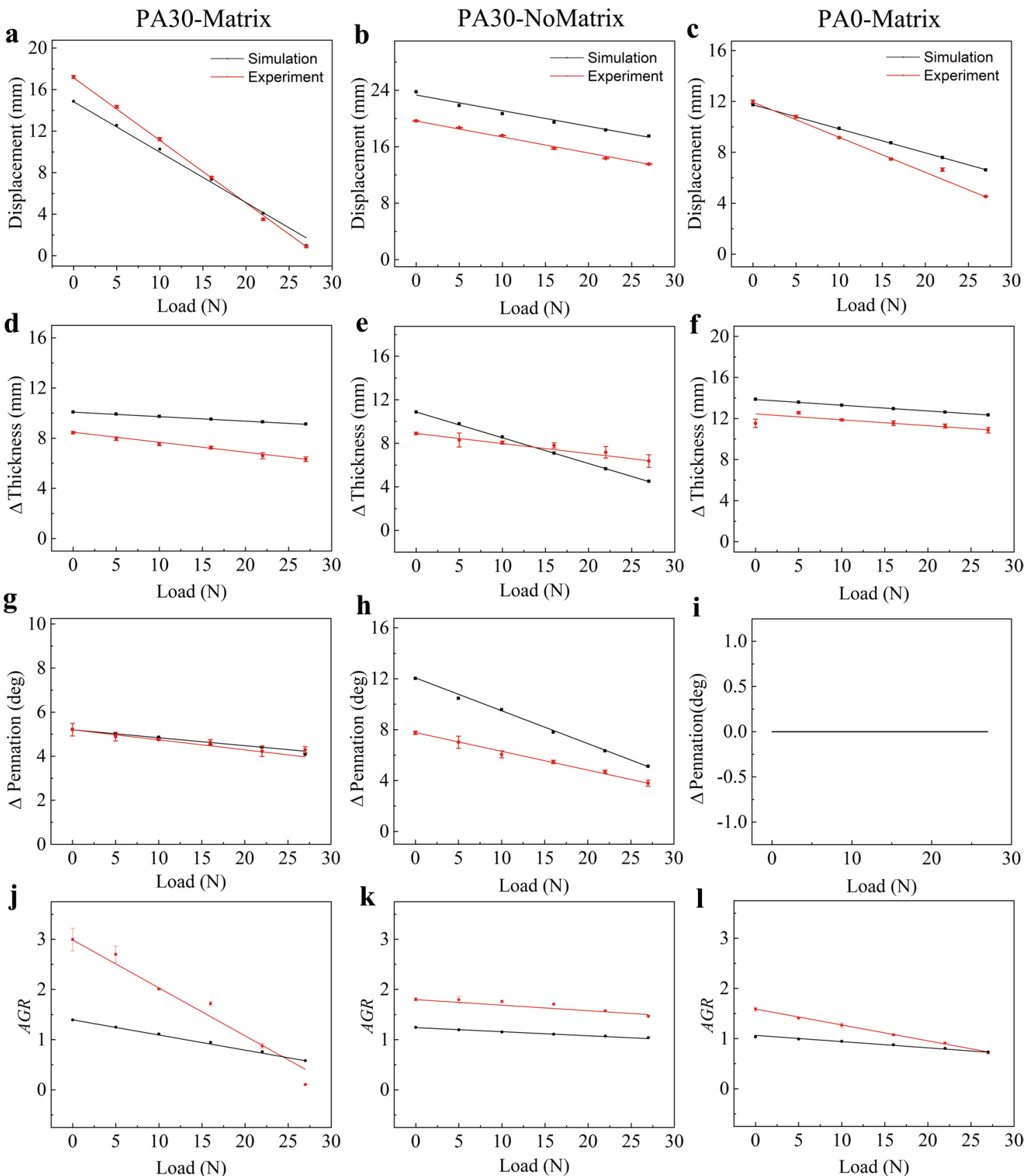

Fig. 5 Comparation between displacement, thickness, and pennation angle of PA30-Matrix, PA30-NoMatrix, and PA0-Matrix actuator in the experiment and simulation. a-c Displacement of PA30-Matrix,

range, this result demonstrated that the variable gearing mechanism presented in the actuator was mediated by the pennation angle and the flexible matrix material. Variable gearing system that is directly integrated to pennate MFUs in an adaptable flexible matrix, which is elegant and simple without gears or clutches compared with conventional ones.
PA30-NoMatrix, and PA0-Matrix actuators. d-f Thickness of three actuators. g-i Pennation angle of three actuators. j-l AGR of three actuators

\subsection{Theoretical Model of Deformation}

To verify the necessity of contractile units and flexible matrix for the gearing mechanism of HimiSK actuator, and illustrate how to achieve beneficial gearing mechanism by their deformation, a theoretical model of the pennate 
Fig. 6 Theory model of HimiSK actuator $\mathbf{a}$

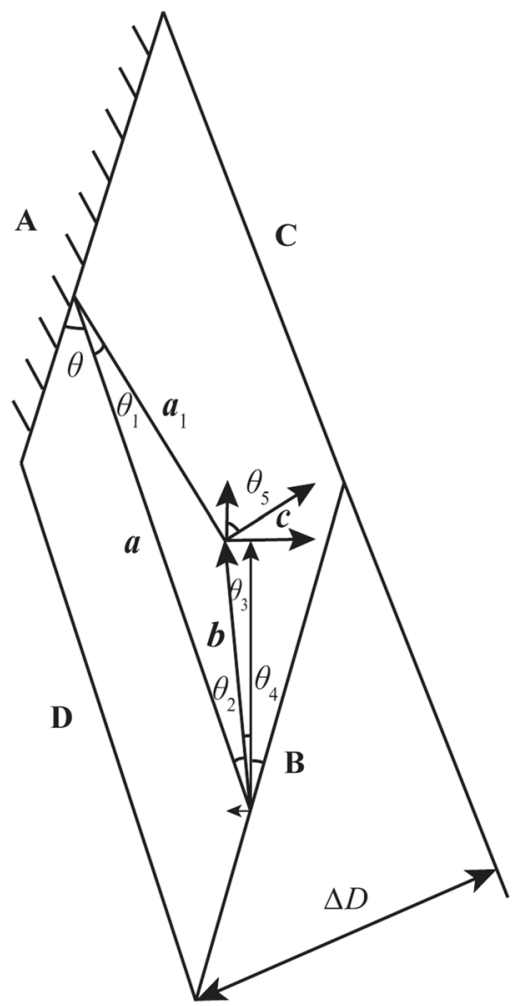

b

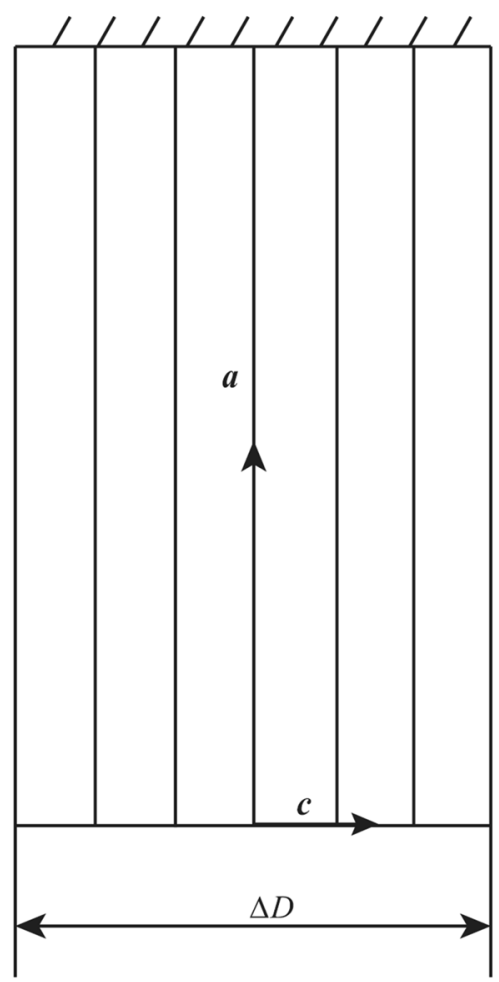

actuator motion is established, as shown in the Fig. 6. As shown in Fig. 6a, the PA30-Matrix actuator is simplified into a parallelogram model. The edge A considers fixed in the process of movement. It decomposes the motion of the contraction unit into axial contraction, radial expansion, and rotational motion. The PA0-Matrix actuator is simplified as a rectangular model (Fig. 6b), which decomposes the movement of the contraction unit into axial contraction and radial expansion.

$\boldsymbol{a}$ is the initial state of the contractile unit, and the effective length is $I_{1}\left(I_{1}=96 \mathrm{~mm}\right) . \boldsymbol{a}_{1}$ is the stable state of contraction and rotation after the contractile unit subjected to air pressure, and its length is $I_{2} \mathrm{~mm}$. $\boldsymbol{b}$ is the displacement of the end of the contractile unit, and length is $I_{3} \mathrm{~mm}$, calculated by formula (1). $\theta$ is the angle between $\mathbf{A}$ and $\boldsymbol{a}$ $\left(\theta=30^{\circ}\right)$, and $\theta_{1}$ is the angle between $\boldsymbol{a}$ and $\boldsymbol{a}_{1}$, which is the angle ( $\Delta$ Pennation) generated during the rotation of contractile unit. The angle between $\boldsymbol{a}$ and $\boldsymbol{b}$ is $\theta_{2}$, obtained by the formula (2). $\theta_{4}$ is the angle between the B-side of the actuator and the vertical direction, $\theta_{4}=15^{\circ}$. The angle of $\theta_{3}$ is obtained. $\theta_{3}=15^{\circ}-\theta_{2}$. The vertical displacement of the contraction unit is $L_{1}, L_{1}=I_{3} \cdot \cos \theta_{3} \mathrm{~mm}$. The horizontal displacement is $T_{1} \cdot T_{1}=I_{3} \cdot \sin \theta_{3} . c$ is the radial expansion of the contractile unit, and its length is $\Delta d \mathrm{~mm}$. Because the contractile units in the actuator is subjected to high pressure, the interference may occur during the expansion of each contraction unit. The deformation of the contractile unit in the expansion direction is calculated by the expansion deformation of the whole actuator. The deformation is $\Delta D$ $\mathrm{mm} . \theta_{5}$ is the angle between the $\mathbf{c}$ and the vertical direction. $\theta_{5}=75^{\circ}-\theta_{1}$. The angle varies with the pinnate angle. The vertical displacement generated during the radial expansion of the contractile unit is $L_{2}, L_{2}=\Delta D \cos \theta_{5}$. A horizonal displacement $T_{2}$ is generated, $T_{2}=\Delta D \sin \theta_{5}$. The total displacement of the actuator is $L$, calculated by formula (3) and the thickness is $T$, calculate by formula (4):

$I_{3}=\sqrt{I_{1}^{2}+I_{2}^{2}-2 I_{1} I_{2}}$,

$\theta_{2}=\arccos \frac{I_{1}^{2}+I_{3}^{2}-I_{2}^{2}}{2 I_{1} I_{3}}$,

$L=L_{1}+L_{2}=I_{3} \cos \theta_{3}+\Delta D \cos \theta_{5}$,

$T=T_{2}-T_{1}=\Delta D \sin \theta_{5}-I_{3} \sin \theta_{3}$.

There is no flexible matrix material in PA30-Nomatrix actuator. Matrix parameters are not affected in deformation. Therefore, the $I_{2}$ and $\Delta D$ of the PA30-Nomatrix actuator are measured first. The deformation blocked coefficient $\gamma$ was obtained at different air pressures after adding the flexible 
Table 3 The parameter of three actuator

\begin{tabular}{llllllll}
\hline Actuator & Pressure & 0 & 0.04 & 0.08 & 0.12 & 0.16 & 0.20 \\
\hline PA30-NoMatrix & $I_{2}$ & 0 & 3.354 & 5.575 & 8.92 & 11.15 & 13.38 \\
& $\Delta D$ & 0 & 2.23 & 5.575 & 8.92 & 11.15 & 13.38 \\
PA30-Matrix & $I_{2}$ & 0 & 3.258 & 4.887 & 8 & 10 & 12.069 \\
& $\Delta D$ & 0 & 2.166 & 4.887 & 8 & 10 & 12.069 \\
PA0-Matrix & $I_{2}$ & 0 & 2.258 & 4.887 & 8 & 11 & 13.069 \\
& $\Delta D$ & 0 & 2.166 & 4.887 & 8 & 10 & 12.069 \\
\hline
\end{tabular}
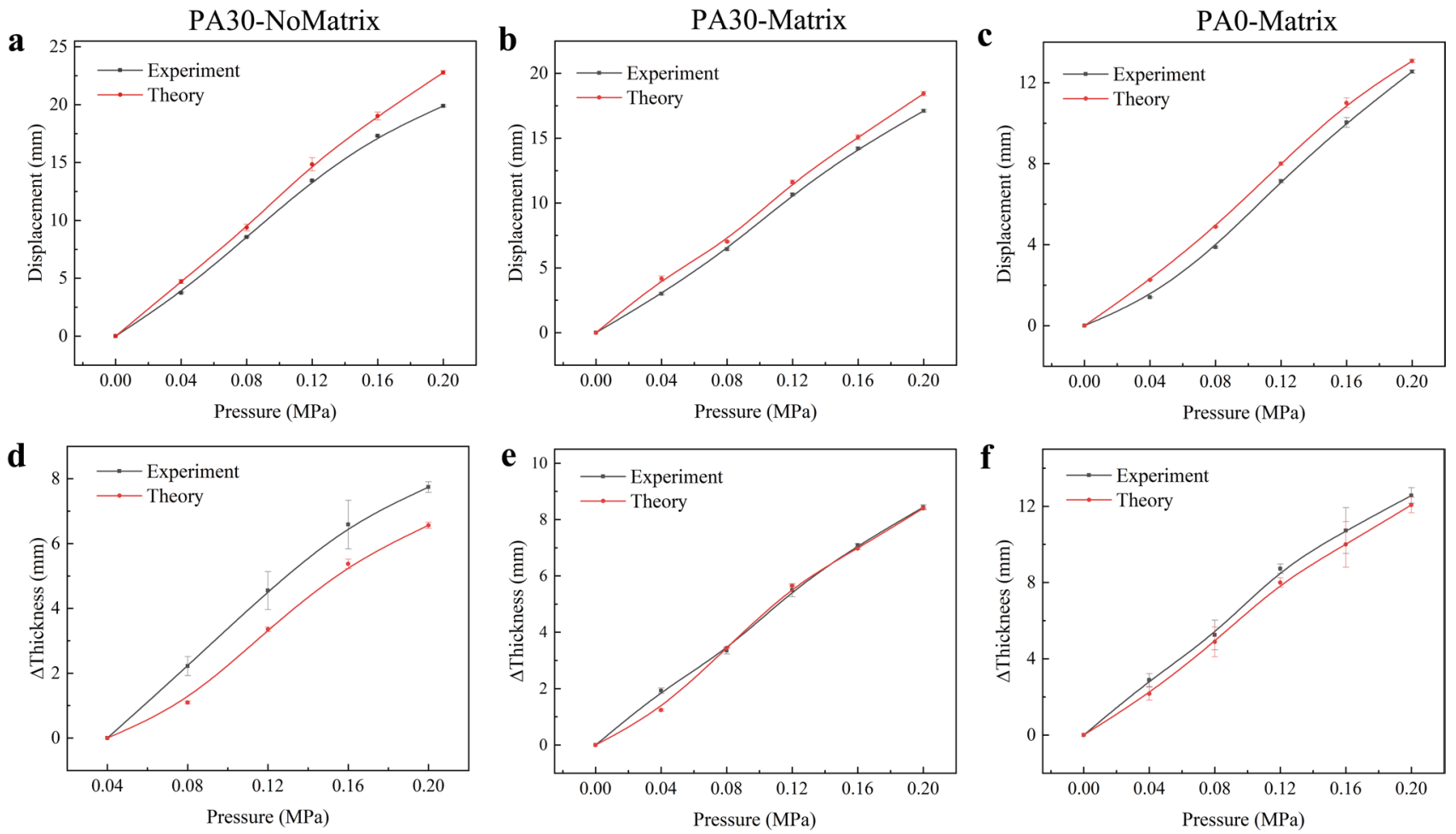

Fig. 7 Comparison of displacement and thickness of three actuators

matrix. The $I_{2}$ and $\Delta D$ of PA30-matrix and PA30-matrix actuator is obtained, as shown in Table 3.

The actuator displacement $L$ and thickness $T$ were compared with the experimental data, under zero load and different pressures $(0,0.04,0.08,0.12,0.16,0.20 \mathrm{MPa})$, as shown in Fig. 7. It can be seen that the displacement and thickness of the three actuators have the same trend with the experiment, and the values are in good agreement. It indicates that the movement of the soft actuator is mainly caused by the axial contraction and radial expansion of the contractile unit. It causes the flexible matrix to deform in the direction of thickness, which drives the contractile unit to rotate and benefit to the contraction of actuator.

It can be clearly seen from Fig.7a-c that the displacement of the pennate actuator (PA30-matrix $(17 \mathrm{~mm})$ ) is higher than that of the parallel actuator (PA0-matrix $(12 \mathrm{~mm})$ ). Although, the pinnate angle reduces the displacement in the vertical component of contractile unit, the displacement of whole actuator in the vertical exceeds the contractile unit, due to the shape changes of flexible matrix of PA30-matrix actuator and the contraction of contractile units. All the contractile units and flexible matrix are the necessary elements for the gearing mechanism. The flexible shape change of soft actuator is a flexible gearing system, its gearing mechanism facilitate shortening length of actuator exceed the shortening length of contractile unit itself, which makes the AGR $>1$. Therefore, the shortening length of the actuator is amplified. Under the same load, the output work of the pennate actuator is larger than that of the non-pennate actuator when AGR $>1$ 


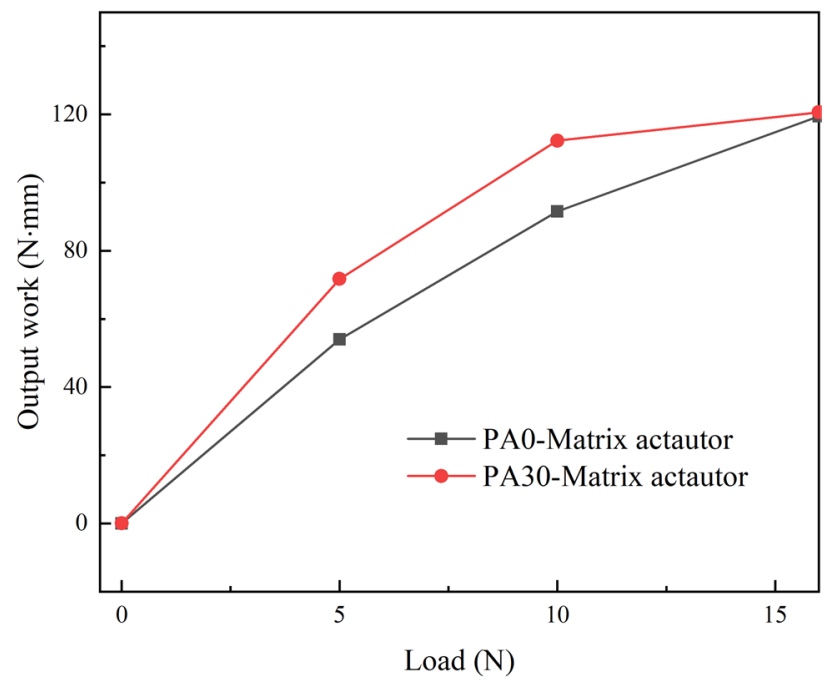

Fig. 8 The output work of PA30-Matrix and PA0-Matrix actuators

(formula (5)), as shown in Fig. 8, Where $F$ respective the output force:

$W_{\text {out }}=F \cdot \Delta L_{\text {actuator }}=\mathrm{AGR} \cdot F \cdot \Delta L_{\mathrm{mfu}}$.

\subsection{AGR at Different Loads Conditions}

We analyzed AGR based on the PA30-Matrix actuators, which with the optimal AGR. When $P=30^{\circ}$ and $N=5$, the $E$ was variable. When $N=5$ and $E=0.6 \mathrm{MPa}$, the $P$ was variable. When $E=0.6 \mathrm{MPa}$ and $P=30^{\circ}$, the $N$ was variable. As shown in Fig. 9, during nearly all contractions, the shortening of the actuator decreased noticeably as the load increasing (Fig.9a, d, g), which was similar to the skeletal muscle. In addition, the shortening of MFUs did not particularly change with loads (Fig. 9b, e, h), which is consistent with the McKibben pneumatic muscle [21, 22]. The contraction of the whole actuator exceeded the contraction of the MFU for all actuators, when AGR $>1$. The calculated AGR significantly decreasing as the load increased at different levels of $P, E$, and $N$ (Fig. 9c, f, i, respectively). This variable gearing mechanism was similar to a phenomenon that has been observed in pennate muscles [14]. The results demonstrate that the dynamic actuator-shape changes promote MFU rotation at low forces and resist MFU rotation at high forces. Therefore, actuator-shape changes act as an automatic transmission system, which can work from a high gear during rapid contractions to a low gear during forceful contractions without the sensory information or feedback control. In addition, the variable gearing mechanism was influenced by the parameter of $P, N$, and $E$. The $E$ effect on AGR is slight but effect on $\Delta L_{\text {actuator }}$ significantly. The analysis provides a better understanding of mechanical behavior of muscle-like soft actuator.

\subsection{AGR with Variable $E, P$, and $N$ at Zero Load}

Further evaluation of the interaction of $P\left(25^{\circ}, 30^{\circ}, 35^{\circ}\right.$, and $\left.40^{\circ}\right) ; N(3,4,5$, and 6); and $E(0.5 \mathrm{MPa}, 0.6 \mathrm{MPa}, 0.7 \mathrm{MPa}$, and $0.8 \mathrm{MPa}$ ), and their influence on AGR was carried out. The 64 sets of simulations are performed under zero load at $0.2 \mathrm{MPa}$ (Fig. 10). Figure 10a-d show the $\Delta L_{\text {actuator }}$ at different $N, P$, and $E$. The $\Delta L$ actuator increased with the $P, E$ decreased, and $N$ increased. When the actuator with a low $P$ and $E$, the MFUs can rotate to a great angle, thus to generate a lager displacement. In addition, since the MFU was the main element of force generation, a larger $N$ could produce a lager displacement as well. In the case of $N$ at 6 and $E$ at $0.5 \mathrm{MPa}$, the displacement was lower, due to a small deformation space between the adjacent contractile units. Figure $10 \mathrm{e}-\mathrm{h}$ reveal the shortening of the contractile unit $\left(\Delta L_{\mathrm{mfu}}\right)$. The $\Delta L_{\mathrm{mfu}}$ increased with $P, N$ increased, and $E$ decreasing. Figure 10i-1 shows the impact of $P$ on the AGR was greatest (from 1.2 to 1.9 , Fig. 10i), and the curves of AGR at different $E$ was similar, because the shortening of contractile units and the actuator were relatively close in the same materials. The results supported the idea that $P$ is the most important factor for the variable gearing mechanism, which is similar to pennate muscle. And the contractile unit was an important factor for variable gearing mechanism as well, due to its capability of force generation. $E$ is a decisive factor affecting displacement/velocity of the actuator but had a negligible influence on the AGR, because the shortening of contractile units and the actuator were relatively close in the same materials. This will provide an effective optimizing strategy for muscle-like soft actuator.

\section{Discussion}

\subsection{Analysis of the Main and Interaction Effects}

To further inspect which factor $(P, E$, or $N)$ was most significant on AGR, three-way ANOVA was performed. The main effects and their interaction effects were illustrated terms of the $F$ value, $p$ value $(p<0.05)$, and partial $\eta^{2}$ value, as shown in Table 4. The analysis results showed that (1) two main effects were significant for AGR: the main effect of $P$ and $N(p<0.001)$. The main effect of $P$ can be interpreted as the average difference between the four $P$ groups $\left(25^{\circ}\right.$, $30^{\circ}, 35^{\circ}$, and $40^{\circ}$ ) across different subgroups (i.e., $N$ and 

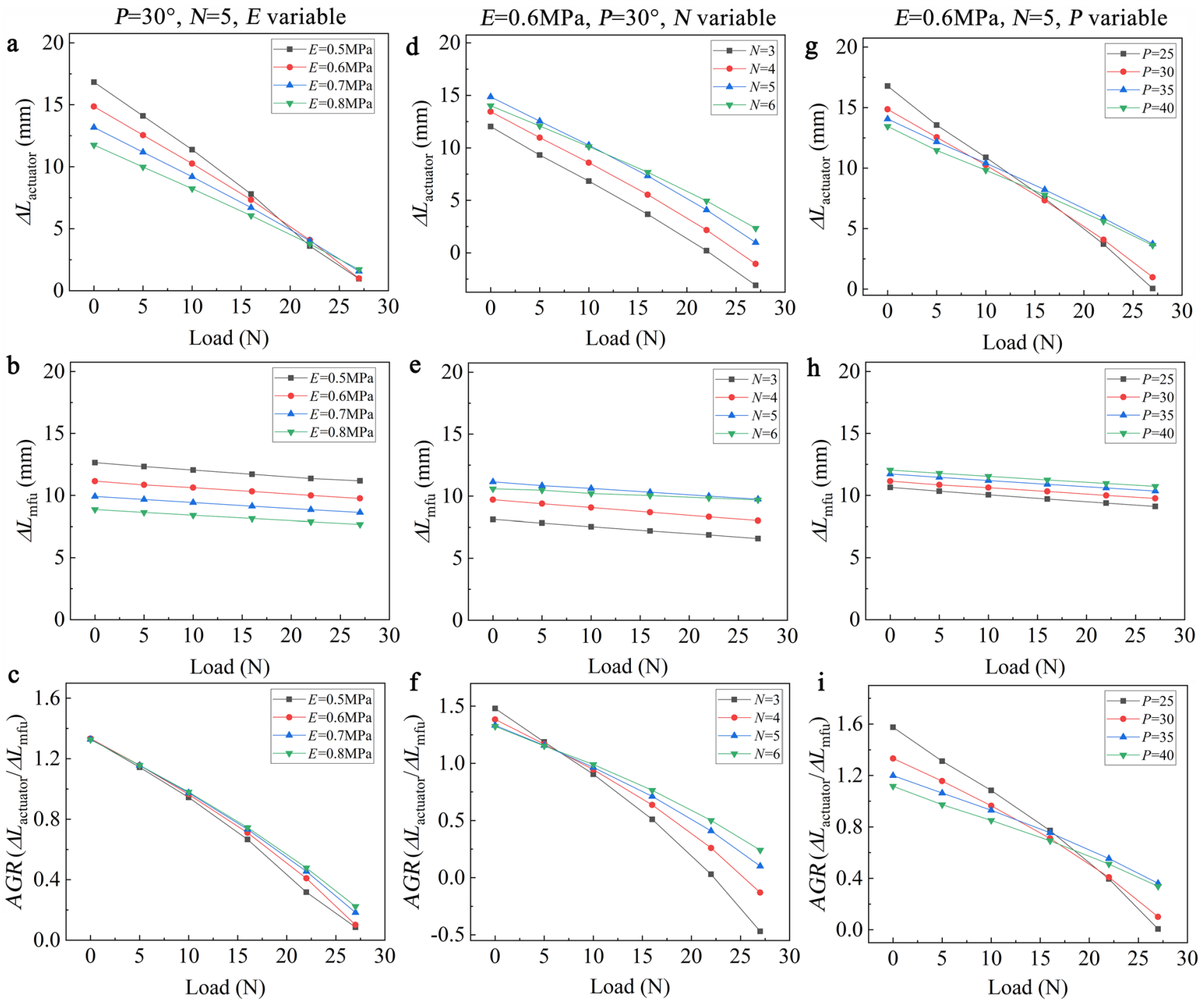

Fig. 9 Changes in actuator, contractile unit displacement, and AGR of loads. a-c Changes in actuator displacement, contractile unit displacement, and AGR when $E$ is variable. $\mathbf{d}-\mathbf{f}$ Changes in the $N$ variable. $\mathbf{g}-\mathbf{i}$ Changes in the $P$ variable

$E$ ). The main effect of $N$ can be interpreted as the average difference between four $N$ groups (3, 4, 5, and 6) across different subgroups (i.e., $P$ and $E$ ). (2) The main effect of $E$ $(p=0.323)$ and the interaction effect of $P$ and $E(p=0.544)$ were not significant. (3) Two interaction effects were significant for AGR: the interaction effect of $P$ and $N(p<0.001)$ and the interaction effect of $E$ and $N(p=0.034)$. The interaction effect of $P$ and $N$ can be interpreted as the difference between four $P$ groups in different $N$ groups. The interaction effect of $E$ and $N$ indicated that there was a significant $N$ effect, and the $N$ effect depended on the condition of the $E$ group. The results suggest that the $P \times N$ and $N \times E$ effects should be investigated in single effect analysis for detailed interpretations.

\subsection{Analysis of the Single Effect and a Comparison}

As shown in Tables 5 and 6 , the single effect analysis revealed significant differences between the interaction of $N$ and $P, E$ and $N$, respectively. Table 5 shows the $F$ value, $p$ value, and partial $\eta^{2}$ of the AGR for the interaction of $N$ and $P$. There was a significant difference $(p<0.001)$ between the single effect of $P$ and $N$. Table 6 shows the $F$ value, $p$ value, and partial $\eta^{2}$ of the AGR for the interaction of $N$ and $E$. There was a significant difference $(p<0.001)$ in the single effect of $N$; but there was no significant difference $(p>0.05)$ in the single effect of $E$, except for in the case of $N=4(p=0.02)$. Partial $\eta^{2}$ in Tables 5 and 6 demonstrate that 

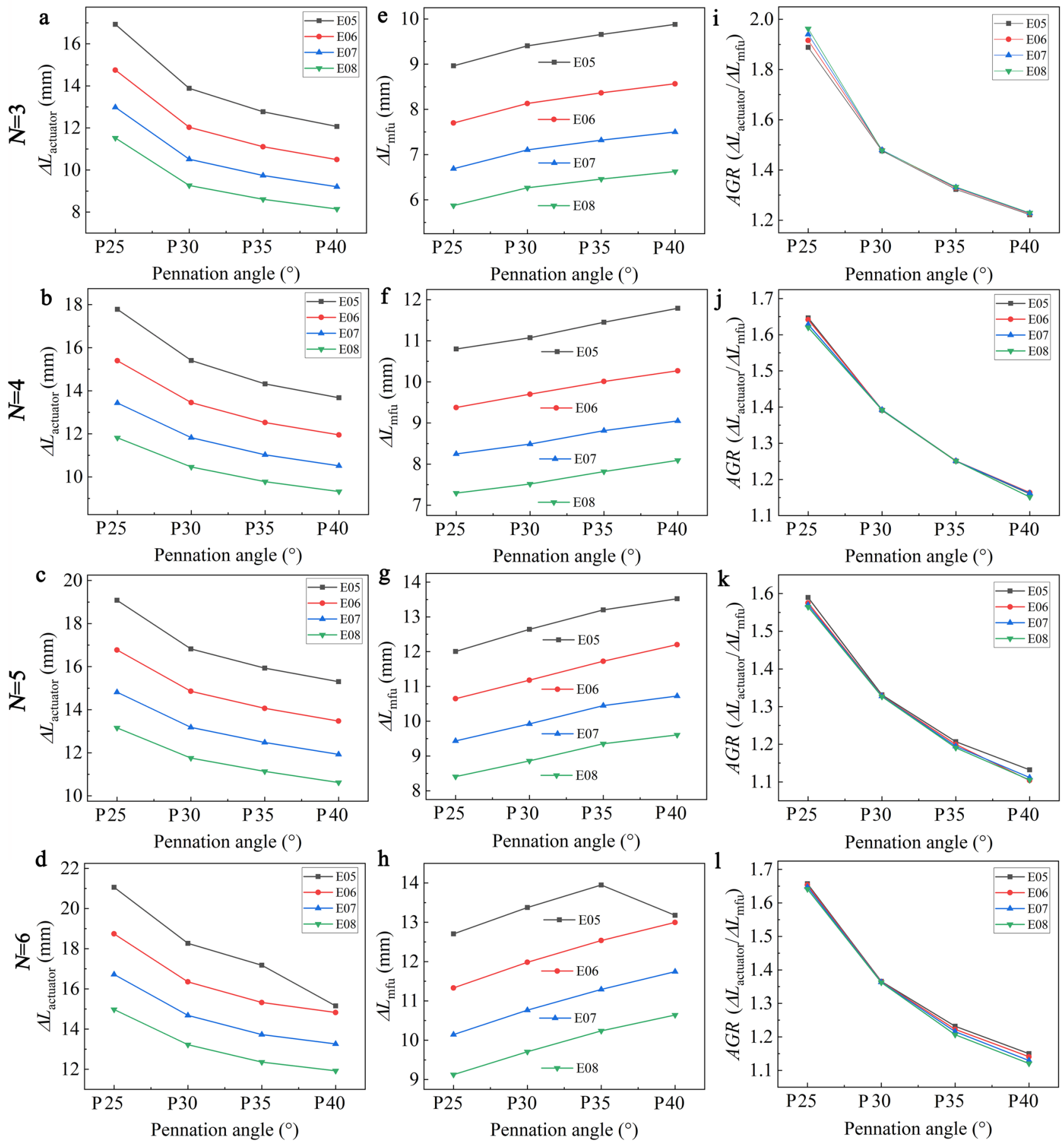

Fig. 10 Changes in AGR of loads at zero loads. a-d Changes in actuator displacement. $\mathbf{e}-\mathbf{h}$ Changes in contractile unit displacement. $\mathbf{i}-\mathbf{l}$ Changes in AGR 
Table $4 F$ value, $p$ value and partial $\eta^{2}$ value with consideration for the interaction

\begin{tabular}{lrrl}
\hline & \multicolumn{1}{l}{$F$} & $p$ & Partial $\eta^{2}$ \\
\hline$N$ & 4644.64 & $<0.001$ & 0.984 \\
$P$ & $31,969.635$ & $<0.001$ & 0.998 \\
$E$ & 15.918 & 0.323 & 0.119 \\
$N \times P$ & 502.316 & $<0.001$ & 0.952 \\
$P \times E$ & 4.621 & 0.544 & 0.230 \\
$N \times E$ & 9.219 & 0.034 & 0.451 \\
\hline
\end{tabular}

the effect of $P$ on AGR was higher than that of other factors ( $E$ and $N$ ), which was similar to the phenomenon that occurs in pennate muscle, in which the pennation angle is the main factor in the variable gearing mechanism. $N$ also influences the AGR due to muscle fiber with the capability of force generation. $E$ has a negligible influence on the AGR.

\section{Conclusion}

In this paper, we presented three types of actuators by varying the arrangement of contractile units and the flexible matrix, termed PA30-Matrix, PA30-NoMatrix, and
PA0-Matrix, respectively. We have demonstrated that all the actuators present load-dependent variable gearing mechanism, and PA30-Matrix actuator with the wide range of AGR. Based on the PA30-Matrix actuator, a series of simulations were performed with a variable pennation angle $(P)$, modulus of elasticity $(E)$, and number of contractile units $(N)$ to investigate their interaction and effects on the variable gearing mechanism. Our study results demonstrated that the $P$ and $N$ significantly affect AGR $(p<0.001)$, individually and in combination $(P \times N$ and $N \times P), E$ has negligible influence on the AGR $(p>0.05)$. The results supported the idea that $P$ is the most important factor for the variable gearing mechanism, which is similar to pennate muscle. The contractile unit was an important factor variable gearing mechanism as well, due to its capability of force generation. $E$ is a decisive factor affecting displacement/velocity of the actuator. The analysis of primary parameters and their interaction for the load-dependent variable gearing mechanism will provide a better understanding of mechanical behavior and an effective optimizing strategy for muscle-like soft actuator.
Table $5 F$ value, $p$ value, and partial $\eta^{2}$ value with consideration for the single effect analysis between $N$ and $P$

\begin{tabular}{llrlllllll}
\hline$N$ & $P$ & $F$ & $p$ & Partial $\eta^{2}$ & $P$ & $N$ & $F$ & $p$ & Partial $\eta^{2}$ \\
\hline 3 & $25,30,35,40$ & 2009.837 & $<0.001$ & 0.996 & 25 & $3,4,5,6$ & 517.489 & $<0.001$ & 0.983 \\
4 & $25,30,35,40$ & 868.719 & $<0.001$ & 0.990 & 30 & $3,4,5,6$ & 86.035 & $<0.001$ & 0.905 \\
5 & $25,30,35,40$ & 852.684 & $<0.001$ & 0.990 & 35 & $3,4,5,6$ & 71.990 & $<0.001$ & 0.889 \\
6 & $25,30,35,40$ & 1073.572 & $<0.001$ & 0.992 & 40 & $3,4,5,6$ & 49.957 & $<0.001$ & 0.847 \\
\hline
\end{tabular}

\begin{tabular}{llllllllll}
\hline$E$ & $N$ & $F$ & $p$ & Partial $\eta^{2}$ & $N$ & $E$ & $F$ & $p$ & Partial $\eta^{2}$ \\
\hline 0.5 & $3,4,5,6$ & 103.575 & $<0.001$ & 0.920 & 3 & $0.5,0.6,0.7,0.8$ & 2.140 & 0.119 & 0.192 \\
0.6 & $3,4,5,6$ & 131.679 & $<0.001$ & 0.936 & 4 & $0.5,0.6,0.7,0.8$ & 3.852 & 0.020 & 0.300 \\
0.7 & $3,4,5,6$ & 149.408 & $<0.001$ & 0.943 & 5 & $0.5,0.6,0.7,0.8$ & 1.293 & 0.297 & 0.126 \\
0.8 & $3,4,5,6$ & 168.735 & $<0.001$ & 0.949 & 6 & $0.5,0.6,0.7,0.8$ & 1.328 & 0.286 & 0.129 \\
\hline
\end{tabular}

Table $6 F$ value, $p$ value, and partial $\eta^{2}$ value with consideration for the single effect analysis between $E$ and $N$ 
Acknowledgements This work was supported by the projects of National Natural Science Foundation of China (No. 52075216, No. 91948302 and No. 91848204)

Author contributions All authors contributed to the study conception and design. Material preparation, data collection and analysis were performed by YW and CL. The first draft of the manuscript was written by YW and all authors commented on previous versions of the manuscript. All authors read and approved the final manuscript.

\section{Declarations}

Conflict of interest The authors declare that there is no conflict of interest.

Ethical approval This article does not contain any studies with human or animal subjects performed by any of the authors.

Open Access This article is licensed under a Creative Commons Attribution 4.0 International License, which permits use, sharing, adaptation, distribution and reproduction in any medium or format, as long as you give appropriate credit to the original author(s) and the source, provide a link to the Creative Commons licence, and indicate if changes were made. The images or other third party material in this article are included in the article's Creative Commons licence, unless indicated otherwise in a credit line to the material. If material is not included in the article's Creative Commons licence and your intended use is not permitted by statutory regulation or exceeds the permitted use, you will need to obtain permission directly from the copyright holder. To view a copy of this licence, visit http://creativecommons.org/licenses/by/4.0/.

\section{References}

1. Biewener, A. A. (2016). Locomotion as an emergent property of muscle contractile dynamics. Journal of Experimental Biology, 219, 285-294.

2. Azizi, E., Brainerd, E. L., \& Roberts, T. J. (2008). Variable gearing in pennate muscles. Proceedings of the National Academy of Sciences of the United States of America, 105, 1745-1750.

3. Brainerd, E. L., \& Azizi, E. (2005). Muscle fiber angle, segment bulging and architectural gear ratio in segmented musculature. Journal of Experimental Biology, 208, 3249-3261.

4. Kovacs, G., Düring, L., Michel, S., \& Terrasi, G. (2009). Stacked dielectric elastomer actuator for tensile force transmission. Sensors and Actuators A: Physical, 155, 299-307.

5. Duduta, M., Hajiesmaili, E., Zhao, H. C., Wood, R. J., \& Clarke, D. R. (2019). Realizing the potential of dielectric elastomer artificial muscles. Proceedings of the National Academy of Sciences of the United States of America, 116, 2476-2481.

6. Kellaris, N., Venkata, V. G., Smith, G. M., Mitchell, S. K., \& Keplinger, C. (2018). Peano-HASEL actuators: Muscle-mimetic, electrohydraulic transducers that linearly contract on activation. Science Robotics, 3, eaar3276.

7. Acome, E., Mitchell, S. K., Morrissey, T. G., Emmett, M. B., Benjamin, C., King, M., Radakovitz, M., \& Keplinger, C. (2018). Hydraulically amplified self-healing electrostatic actuators with muscle-like performance. Science, 359, 61-65.

8. Yang, H., Xu, M., Li, W. H., \& Zhang, S. W. (2018). Design and implementation of a soft robotic arm driven by SMA coils. IEEE Transactions on Industrial Electronics, 66, 6108-6166.
9. Zhang, J. J., \& Yin, Y. H. (2012). SMA-based bionic integration design of self-sensor-actuator-structure for artificial skeletal muscle. Sensors and Actuators A: Physical, 181, 94-102.

10. Villanueva, A., Smith, C., \& Priya, S. (2011). A biomimetic robotic jellyfish (Robojelly) actuated by shape memory alloy composite actuators. Bioinspiration and Biomimetics, 6, 036004.

11. Kanik, M., Orguc, S., Varnavides, G., Kim, J., Benavides, T., Gonzalez, D., Akintilo, T., Tasan, C. C., Chandrakasan, A. P., Fink, Y., \& Anikeeva, P. (2019). Strain-programmable fiber-based artificial muscle. Science, 365, 145-150.

12. Lee, J. A., Li, N., Haines, C. S., Kim, K. J., Lepró, X., OvalleRobles, R., Kim, S. J., \& Baughman, R. H. (2017). Electrochemically powered, energy-conserving carbon nanotube artificial muscles. Advanced Materials, 29, 1700870.

13. Mu, J. K., de Andrade, M. J., Fang, S. L., Wang, X. M., Gao, E. L., Li, N., Kim, S. H., Wang, H. Z., Hou, C. Y., Zhang, Q. H., Zhu, M. F., Qian, D., Lu, H. B., Kongahage, D., Talebian, S., Foroughi, J., Spinks, G., Kim, H., Ware, T. H., ... Baughman, R. H. (2019). Sheath-run artificial muscles. Science, 365, 150-155.

14. Azizi, E., \& Roberts, T. J. (2013). Variable gearing in a biologically inspired pneumatic actuator array. Bioinspiration and Biomimetics, 8, 026002.

15. Roche, E. T., Horvath, M. A., Wamala, I., Alazmani, A., Song, S. E., Whyte, W., Machaidze, Z., Payne, C. J., Weaver, J. C., Fishbein, G., Kuebler, J., Vasilyev, N. V., Mooney, D. J., Pigula, F. A., $\&$ Walsh, C. J. (2017). Soft robotic sleeve supports heart function. Science Translational Medicine, 9, eaaf3925.

16. Li, S., Vogt, D. M., Rus, D., \& Wood, R. J. (2017). Fluid-driven origami-inspired artificial muscles. Proceedings of the National Academy of Sciences of the United States of America, 114, 13132-13137.

17. Yang, D., Verma, M. S., So, J.-H., Mosadegh, B., Keplinger, C., Lee, B., Khashai, F., Lossner, E., Suo, Z., \& Whitesides, G. M. (2016). Buckling pneumatic linear actuators inspired by muscle. Advanced Materials Technologies, 1, 1600055.

18. Roche, E. T., Wohlfarth, R., Overvelde, J. T., Vasilyev, N. V., Pigula, F. A., Mooney, D. J., Bertoldi, K., \& Walsh, C. J. (2014). A bioinspired soft actuated material. Advanced Materials, 26, 1200-1206.

19. Diteesawat, R. S., Helps, T., Taghavi, M., \& Rossiter, J. (2020). Characteristic analysis and design optimization of bubble artificial muscles. Soft Robotics, 8, 186-199.

20. Klute, G. K., Czerniecki, J. M., \& Hannaford, B. (2002). Artificial muscles: Actuators for biorobotic systems. International Journal of Robotics Research, 21, 295-309.

21. Chou, C. P., \& Hannaford, B. (1996). Measurement and modeling of McKibben pneumatic artificial muscles. Robotics and Automation, IEEE Transactions on Robotics and Automation, 12, 90-102.

22. Klute, G. K., Czerniecki, J. M., \& Hannaford, B. (1999). McKibben artificial muscles: Pneumatic actuators with biomechanical intelligence. 1999 IEEE/ASME International Conference on Advanced Intelligent Mechatronics (Cat. No.99TH8399), Atlanta, USA, 1999, 221-226.

23. Roberts, T. J., Eng, C. M., Sleboda, D. A., Holt, N. C., Brainerd, E. L., Stover, K. K., Marsh, R. L., \& Azizi, E. (2019). The multiscale, three-dimensional nature of skeletal muscle contraction. Physiology, 34, 402-408.

24. Azizi, E., \& Roberts, T. J. (2014). Geared up to stretch: Pennate muscle behavior during active lengthening. Journal of Experimental Biology, 217, 376-381.

25. Gao, Y. X., \& Zhang, C. (2015). Structure-function relationship of skeletal muscle provides inspiration for design of new artificial muscle. Smart Materials and Structures, 24, 033002. 
26. Holt, N. C., Danos, N., Roberts, T. J., \& Azizi, E. (2016). Stuck in gear: Age-related loss of variable gearing in skeletal muscle. The Journal of Experimental Biology, 219, 998-1003.

27. Eng, C. M., Emanuel, A., \& Roberts, T. J. (2018). Structural determaints of muscle gearing during dynamic contractions. Integrative and Comparative Biology, 58, 207-217.

28. Polygerinos, P., Correll, N., Morin, S. A., Mosadegh, B., Onal, C. D., Petersen, K., Cianchetti, M., Tolley, M. T., \& Shepherd, R. F. (2017). Soft robotics: Review of fluid-driven intrinsically soft devices; manufacturing, sensing, control, and applications in human-robot interaction. Advanced Engineering Materials, 19, 1700016.

Publisher's Note Springer Nature remains neutral with regard to jurisdictional claims in published maps and institutional affiliations. 